\title{
White Matter/Gray Matter Contrast Changes in Chronic and Diffuse Traumatic Brain Injury
}

\author{
Eva M. Palacios, ${ }^{1, \star}$ Roser Sala-Llonch,, ${ }^{1, \star}$ Carme Junque, ${ }^{1}$ Teresa Roig, ${ }^{2}$ Jose M. Tormos, ${ }^{2}$ \\ Nuria Bargallo, ${ }^{3}$ and Pere Vendrell ${ }^{1}$
}

\begin{abstract}
Signal-intensity contrast of T1-weighted magnetic resonance imaging scans has been associated with tissue integrity and reported as a sign of neurodegenerative changes in diseases such as Alzheimer's disease. After severe traumatic brain injury (TBI), progressive structural changes occur in white (WM) and gray matter (GM). In the current study, we assessed the signal-intensity contrast of GM and WM in patients with diffuse TBI in the chronic stage to (1) characterize the regional pattern of WM/GM changes in intensity contrast associated with traumatic axonal injury, (2) evaluate possible associations between this measure and diffusion tensor image (DTI)/fractional anisotropy (FA) for detecting WM damage, and (3) investigate the correlates of both measures with cognitive outcomes. Structural T1 scans were processed with FreeSurfer software to identify the boundary and calculate the WM/GM contrast maps. DTIs were processed with the FMRIB software library to obtain FA maps. The WM/GM contrast in TBI patients showed a pattern of reduction in almost all of the brain, except the visual and motor primary regions. Global FA values obtained from DTI correlated with the intensity contrast of all associative cerebral regions. WM/GM contrast correlated with memory functions, whereas FA global values correlated with tests measuring memory and mental processing speed. In conclusion, tissue-contrast intensity is a very sensitive measure for detecting structural brain damage in chronic, severe and diffuse TBI, but is less sensitive than FA for reflecting neuropsychological sequelae, such as impaired mental processing speed.
\end{abstract}

Key words: cognitive deficits; diffusion tensor imaging (DTI); traumatic brain injury; white matter/gray matter signalintensity contrast

S TRUCTURAL BRAIN DAMAge in white and gray matter (WM/GM) can be observed and quantified by magnetic resonance imaging (MRI) after chronic traumatic brain injury (TBI). This damage is responsible for cognitive impairment and daily life disability. ${ }^{1}$ Traumatic axonal injury (TAI) mainly causes WM damage reflecting primary and secondary axotomy. ${ }^{2}$ Further, progressive retrograde degeneration resulting from TAI may also be the cause of neuronal loss and the resulting GM changes. ${ }^{3}$ Diffusion tensor imaging (DTI) is the technique of choice for detecting WM damage alterations. These alterations have been reported to be the main cause of cognitive impairment after TBI with diffuse pathology in the chronic stage. ${ }^{3-5}$ In the current study, we assessed the contrast between WM/GM signal intensities in patients with severe diffuse TBI in the chronic stage and a group of controls and compared it with DTI/fractional anisotropy (FA), the main indicator of WM integrity. The contrast between WM/GM signal intensities extracted from T1-weighted $\mathrm{MRI}^{7}$ may have advantages over DTI, given that this sequence has a greater resolution, shorter scanning time, which may facilitate clinical use, and the fact that GM changes are also taken into account. This measure has also been related to tissue integrity and myelin degradation. ${ }^{6}$ Therefore, the aims of the study were to (1) characterize the regional pattern of WM/GM differences associated with TAI, (2) evaluate possible relationships between this measure and DTI-FA in the detection of WM damage, and (3) investigate the relationship of the two measures with cognitive outcome.

The TBI group included 26 patients with a mean age of 27.40 $( \pm 5.15)$ years. Patients were examined at a mean time of evolution postinjury of $4.20( \pm 1.14)$ years and a mean degree of severity measured by the Glasgow Coma Scale of 5.19 ( \pm 1.70$)$. The main brain alterations observed in the scans (T2/FLAIR sequences) were microbleeds as a sign of diffuse pathology without significant

\footnotetext{
${ }^{1}$ Department of Psychiatry and Clinical Psychobiology, University of Barcelona, Barcelona, Spain.

${ }^{2}$ Institute Guttmann, Neurorehabilitation Hospital, Barcelona, Spain.

${ }^{3}$ Centre de Diagnostic per la Imatge Hospital Clinic de Barcelona (CDIC), Hospital Clinic de Barcelona, Spain.

*The first two authors contributed equally.
} 
contusions. The criteria applied for the selection and characteristics of the sample have been described elsewhere. ${ }^{3}$ The control group comprised 22 healthy volunteers matched by age (mean, 28.57 $[ \pm 6.74])$, sex, handedness, and education. ${ }^{3}$ MRI data were acquired on a Siemens MAGNETOM Trio, A Tim System 3T (Siemens AG, Munich, Germany) at the Diagnostic Imaging Center of the Hospital Clinic in Barcelona, Spain. A high-resolution T1weighted structural image scan was obtained for each subject using an MPRAGE three-dimensional protocol (resolution time [TR]= $2300 \mathrm{msec}$; echo time $[\mathrm{TE}]=3 \mathrm{msec}$; inversion time $[\mathrm{TI}]=900$ msec; field of view $[\mathrm{FOV}]=244 \mathrm{~mm} ; 1 \mathrm{~mm}$ isotropic voxel). Diffusion MRI (dMRI) data were acquired in the same session in 30 noncollinear directions with a b-value $=1000 \mathrm{~s} / \mathrm{mm}^{2}$, using an echo-planar imaging (EPI) sequence $(\mathrm{TR}=9300 \mathrm{~ms}$; $\mathrm{TE}=94 \mathrm{~ms}$; slice thickness $=2.0 \mathrm{~mm}$; voxel size $=2.0 \times 2.0 \times 2.0 \mathrm{~mm}$; FOV $=$ $240 \mathrm{~mm}^{2}$, no gap).

Structural T1 scans were processed with FreeSurfer software (http://surfer.nmr.mgh.harvard.edu/), which performs automated tissue segmentation, identification of the WM/GM boundary and pial surface, estimation of cortical thickness maps, and interindividual spherical registration. ${ }^{8,9}$ Intensity maps for WM and GM were created using the values of the T1-weighted signal of the WM at $1 \mathrm{~mm}$ below the white surface, and the values of the GM were taken at half of the thickness of the cortex. Finally, WM/GM intensity contrast maps were calculated using the percentage contrast between WM and GM intensities. Before performing group statistical analyses, the resulting WM, GM, and WM/GM contrast maps were mapped to a common surface and smoothed with a 15mm Gaussian kernel of full width at half maximum. FA maps were obtained individually from dMRI data and registered together using FMRIB software library (FSL) software (http://fsl.fmrib.ox.ac.uk/ fsl/fslwiki/FSL). All FA maps were further projected to a common skeleton, which represents the main tracts within all the subjects, using tract-based spatial statistics. ${ }^{10}$ Finally, global FA scores for each subject were calculated as the average FA within the skeleton, and these values were used as a measure for whole-brain connectivity.

Differences between controls and patients in the WM/GM contrast maps were tested in a voxel-wise analysis using a general linear model and corrected for multiple comparisons using $\mathrm{Z}$ Monte Carlo simulations (10,000 iterations). Cluster threshold for significance was set at $p<0.05$. To measure the influence of GM and WM intensities on the results of the GM/WM contrast, the same statistical analyses were performed independently on GM and WM surface maps. Average values within the entire cortex were obtained for the WM/GM contrast as well as for WM and GM intensities. Moreover, to test the role of WM integrity changes in explaining the WM/GM intensity contrast, a correlation between this measurement and global FA values was also undertaken.

We found that TBI patients performed significantly worse than the control group in all the neuropsychological tests (Table 1, A). To check the normality of our data, we used Shapiro-Wilk's test. Pearson's correlation was used for data that followed normality. When data did not follow a normal distribution, nonparametric tests were used to compare groups (Mann-Whitney's U) and to perform correlations (Spearman's correlation). A reduction in WM/GM contrast was observed in almost all of the brain, except for the visual and motor primary regions (Fig. 1A). Global values of WM/ GM contrast were significantly reduced in TBI (Table 1, A). Global FA values correlated with the intensity contrast of all associative cerebral regions, with a predominance of the prefrontal cortex (Fig. 1B). Considering whole-brain measures, global FA correlated with global WM/GM contrast for patients $(r=0.605 ; p=0.001)$. In TBI patients, global WM/GM intensity contrast values correlated with memory functions and global FA values correlated with tests measuring memory and mental processing speed (Table 1, B). None of these correlations were found in the control group.

Table 1. Comparison of Neuropsychological Performance between Patients and Controls and Correlations of the Tests with GM/WM Intensity Contrast and FA Values

\begin{tabular}{|c|c|c|c|c|c|}
\hline & \multicolumn{3}{|c|}{ A. Group comparison } & \multicolumn{2}{|c|}{ B. Correlations with neuroimaging variables $T B I$} \\
\hline & $\begin{array}{l}\text { TBI group } \\
(\text { mean/SD) }\end{array}$ & $\begin{array}{l}\text { Control group } \\
(\text { mean/SD) }\end{array}$ & $\begin{array}{l}\text { Statistic } \\
\mathrm{t}(\mathrm{p})\end{array}$ & $\begin{array}{l}\text { Whole-brain WM/GM } \\
\text { intensity statistic } \mathrm{r}(\mathrm{p})\end{array}$ & $\begin{array}{l}\text { Mean whole-FA } \\
\text { statistic } \mathrm{r}(\mathrm{p})\end{array}$ \\
\hline WM/GM intensity & $22.41( \pm 1.38)$ & $24.43( \pm 1.15)$ & $5.43(<0.001)^{*}$ & - & $0.6(0.001)^{*}$ \\
\hline RAVLT learning & $45.42( \pm 10.26)$ & $60.10( \pm 5.86)$ & $5.93(0.01)$ & $0.61(0.001)^{*}$ & $0.53(0.005)^{*}$ \\
\hline RAVLT (DR) & $7.81( \pm 3.14)$ & $13.50( \pm 1.56)$ & $7.72(0.002)^{*}$ & $0.62(0.001)^{*}$ & $0.59(\leq 0.001)^{*}$ \\
\hline Digits forward & $8.67( \pm 1.71)$ & $10.18( \pm 1.86)$ & $2.29(0.02)^{a}$ & $-0.28(0.16)^{a}$ & $-0.14(0.47)^{\mathrm{a}}$ \\
\hline Digits backward & $6.19( \pm 1.44)$ & $7.50( \pm 1.47)$ & $3.10(0.03)$ & $0.07(0.73)$ & $0.05(0.78)$ \\
\hline LNS & $9.69( \pm 2.07)$ & $12.48( \pm 1.05)$ & $5.32(<0.001)^{*}$ & $0.36(0.07)$ & $0.21(0.29)$ \\
\hline TMTA & $41.73( \pm 31.30)$ & $22.73( \pm 6.07)$ & $3.02(0.05)$ & $-0.31(0.12)$ & $-0.47(0.01)$ \\
\hline ТМТВ & $101.38( \pm 80.38)$ & $55.45( \pm 14,05)$ & $2.86(0.008)$ & $-0.27(0.15)$ & $-0.45(0.02)$ \\
\hline TMTB-A & $59.65( \pm 52.90)$ & $33.09( \pm 15.08)$ & $2.44(0.021)$ & $-0.24(0.22)$ & $-0.39(0.04)$ \\
\hline Symbol Digit & $68.04( \pm 15,64)$ & $87.18( \pm 15.53)$ & $4.24(<0.001)^{*}$ & $0.25(0.23)$ & $0.33(0.06)$ \\
\hline Stroop (WR) & $96.61( \pm 16.68)$ & $109.00( \pm 12.65)$ & $2.76(0.09)$ & $-0.09(0.67)$ & $0.09(0.67)$ \\
\hline Stroop $(\mathrm{CN})$ & $63.91( \pm 9.01)$ & $76.65( \pm 9.14)$ & $4.58(<0.001)^{*}$ & $-0.12(0.59)$ & $0.017(0.94)$ \\
\hline Stroop (CWN) & $44.83( \pm 9.42)$ & $51.70( \pm 7.51)$ & $2.65(0.01)$ & $0.04(0.83)$ & $-0.07(0.72)$ \\
\hline Semantic fluency & $19.08( \pm 6.65)$ & $24.43( \pm 4.79)$ & $3.15(0.003)$ & $0.25(0.22)$ & $0.33(0.10)$ \\
\hline Phonetic fluency & $33.38( \pm 12.58)$ & $45.86( \pm 10.15)$ & $3.75(<0.001)^{*}$ & $0.33(0.09)$ & $0.21(0.30)$ \\
\hline
\end{tabular}

${ }^{\mathrm{a}}$ Nonparametric tests were used for comparisons between groups and correlations.

"Statistical significance after Bonferroni's correction for multiple comparisons (corrected $p<0.05$ ).

GM, gray matter; WM, white matter; FA, fractional anisotropy; TBI, traumatic brain injury; SD, standard deviation; RAVLT, Rey Auditory-Verbal Learning Test; DR, Delayed Recall; LNS, Letter-Number Sequencing; TMT, Trail Making Test; WR, Word Reading; CN, Color Naming; CWN, ColorWord Naming. 


\section{A INTENSITY GM/WM GROUP COMPARISON}

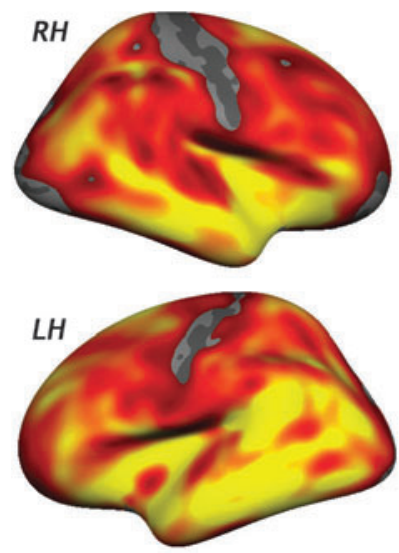

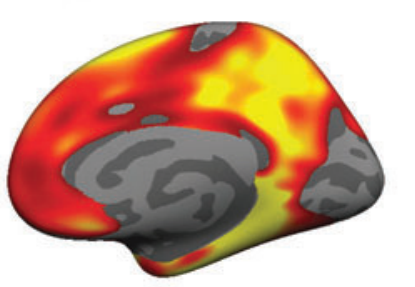

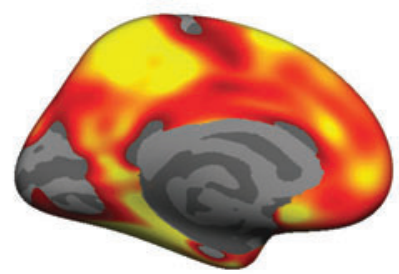

B CORRELATIONS BETWEEN GM/WM INTENSITY \& FA
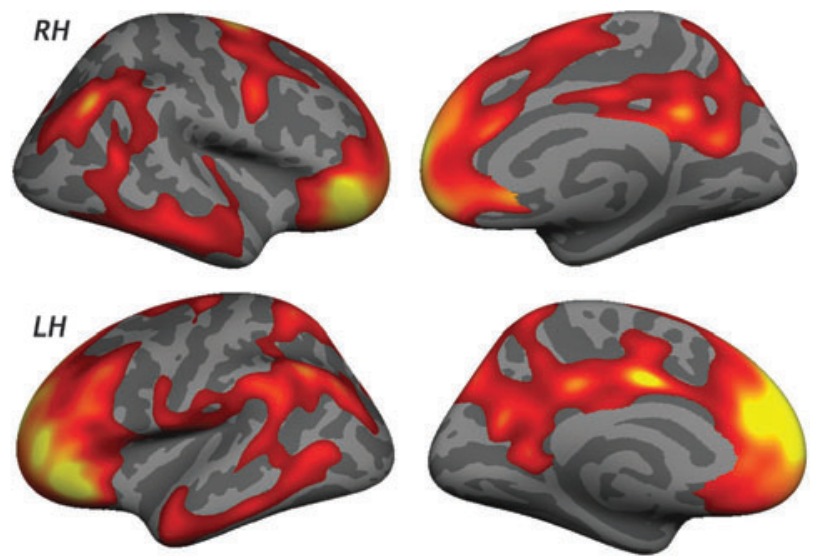

$p$ value

FIG. 1. Results of the statistical tests on GM/WM contrast maps after correction for multiple comparisons. Raw $p$ values were used to show the areas with greater effects. (A) Results of the group comparison between patients and controls. (B) Results of the correlation between GM/ WM contrast and global FA values. All resulting clusters had a corrected cluster-wise $p$ value of 0.0001. GM, gray matter; WM, white matter; FA, fractional anisotropy; RH, right hemisphere; LH, left hemisphere. Color image is available online at www.liebertpub.com/neu

To the best of our knowledge, this is the first study to assess WM/ GM signal-intensity contrast and its association with cognitive impairment in patients with diffuse TBI in the chronic stage. The group comparison results showed that chronic TBI patients had a pattern of decreased WM/GM contrast in all of the brain, with the exception of the visual and motor primary regions. Global measures of WM/GM contrast corroborated this result. Unlike the independent comparison of WM and GM intensity maps, this finding shows WM/GM signalintensity contrast to be a highly sensitive measure for the detection of structural sequelae in TBI patients. Significant brain changes were observed in an earlier study by our group of the same sample using cortical thickness, ${ }^{3}$ but these were brought into even greater relief using WM/GM signal intensity, presumably as a result of the inclusion of WM alterations in the measurement. In other pathologies, such as Alzheimer's disease, the WM/GM intensity contrast has also been reported as being more sensitive to distinct brain pathology than cortical thickness. ${ }^{6}$ Because our sample comprised subjects with evidence of TAI, the main brain damage was produced in the WM. However, GM may also have been impaired as a result of retrograde and transneuronal degeneration. ${ }^{11}$

On relating global FA values with WM/GM intensity contrast, it was found that the decrease in global FA explained the loss of intensity contrast measure in the associative regions of the cortex, particulary in the heteromodal association cortex. Studies of wholebrain WM connectivity using graph theory in healthy subjects have defined several cortical hubs in the associative cortex, which are believed to manage an integrative brain function. These regions show a far greater number of connections, in comparison with primary regions, ${ }^{12}$ and it is presumably this greater connectivity that leads to the more widespread WM damage and greater impairment. ${ }^{13,14}$

With regards to the correlations with neuropsychological sequelae, we observed that memory impairment was related with both global measures (WM/GM and FA), but that only global FA, a purer measure of WM integrity, was associated with mental processing speed impairments.
The diffuse and chronic characteristics of our sample may preclude the generalization of the results to TBI patients with focal lesions and patients in acute or postacute stages. Longitudinal studies in acute patients using these more sensitive measures are needed to understand and predict the dynamic changes, such as the progression of neurodegenerative structural damage and its reorganization, that will help to explain the evolution of cognitive impairments after TBI.

\section{Acknowledgments}

The authors thank Cesar Garrido from the Diagnostic Imaging Center of the Hospital Clinic (CDIC) in Barcelona for assistance with data recollection.

\section{Author Disclosure Statement}

No competing financial interests exist.

\section{References}

1. Benedictus, M.R., Spikman, J.M., and van der Naalt, J. (2010). Cognitive and behavioral impairment in traumatic brain injury related to outcome and return to work. Arch. Phys. Med. Rehabil. 91, 1436-1441.

2. Büki, A., and Povlishock, J.T. (2006). All roads lead to disconnection?Traumatic axonal injury revisited. Acta Neurochir. 148, 181-193.

3. Palacios, E.M., Sala-Llonch, R., Junque, C., Fernandez-Espejo, D., Roig, T., Tormos, J.M., Bargallo, N., and Vendrell, P. (2013). Longterm declarative memory deficits in diffuse TBI: correlations with cortical thickness, white matter integrity and hippocampal volume. Cortex 49, 646-657.

4. Palacios, E.M., Sala-Llonch, R., Junque, C., Roig, T., Tormos, J.M., Bargallo, N., and Vendrell, P. (2012). White matter integrity related to functional working memory networks in traumatic brain injury. Neurology 78, 852-860.

5. Kraus, M.F., Susmaras, T., Caughlin, B.P., Walker, C.J., Sweeney, J.A., and Little, D.M. (2007). White matter integrity and cognition in chronic traumatic brain injury: a diffusion tensor imaging study. Brain 130, 2508-2519.

6. Salat, D.H., Lee, S.Y., van der Kouwe, A.J., Greve, D.N., Fischl, B., and Rosas, H.D. (2009). Age-associated alterations in cortical gray 
and white matter signal intensity and gray to white matter contrast. Neuroimage 48, 21-28.

7. Grydeland, H., Westlye, L.T., Walhovd, K.B., and Fjell, A.M. (2012). Improved prediction of Alzheimer's disease with longitudinal white matter/gray matter contrast changes. Hum. Brain Mapp. Jun 5. doi: 10.1002/hbm.22103. [Epub ahead of print].

8. Dale, A.M., Fischl, B., and Sereno, M.I. (1999). Cortical surface-based analysis. I. Segmentation and surface reconstruction. Neuroimage 9, 179-194.

9. Fischl, B., and Dale, A.M. (2000). Measuring the thickness of the human cerebral cortex from magnetic resonance images. Proc. Natl. Acad. Sci. U. S. A. 97, 11050-11055.

10. Smith, S.M., Jenkinson, M., Johansen-Berg, H., Rueckert, D., Nichols, T.E., Mackay, C.E., Watkins, K.E., Ciccarelli, O., Cader, M.Z., Matthews, P.M., and Behrens, T.E.J. (2006). Tract-based spatial statistics: voxelwise analysis of multi-subject diffusion data. Neuroimage 31, 1487-1505.

11. Maxwell, W.L., MacKinnon, M.A., Stewart, J.E., and Graham, D.I. (2010). Stereology of cerebral cortex after traumatic brain injury matched to the Glasgow outcome score. Brain 133, 139-60.
12. Gong, G., He, Y., Concha, L., Lebel, C., Gross, D.W., Evans, A.C., and Beaulieu, C. (2009). Mapping anatomical connectivity patterns of human cerebral cortex using in vivo diffusion tensor imaging tractography. Cereb. Cortex 19, 524-536.

13. Honey, C.J., and Sporns, O. (2008). Dynamical consequences of lesions in cortical networks. Hum. Brain Mapp. 29, 802-9.

14. Alstott, J., Breakspear, M., Hagmann, P., Cammoun, L., and Sporns, O. (2009). Modeling the impact of lesions in the human brain. PLoS Comput. Biol. 5:e1000408.

Address correspondence to:

Carme Junque, PhD

Department of Psychiatry and Clinical Psychobiology

University of Barcelona

C/Casanova 143

08036 Barcelona, Spain

E-mail: cjunque@ub.edu 